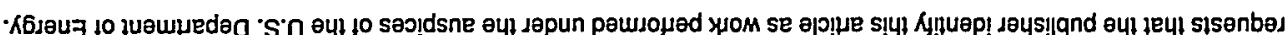

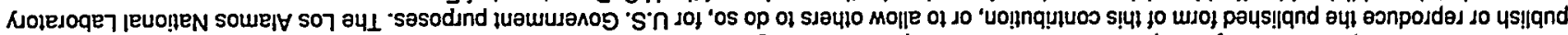

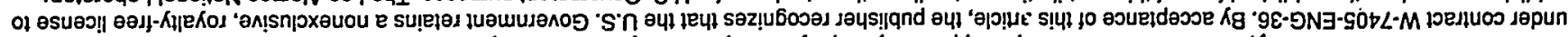

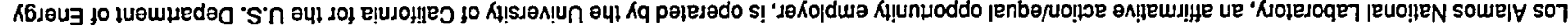

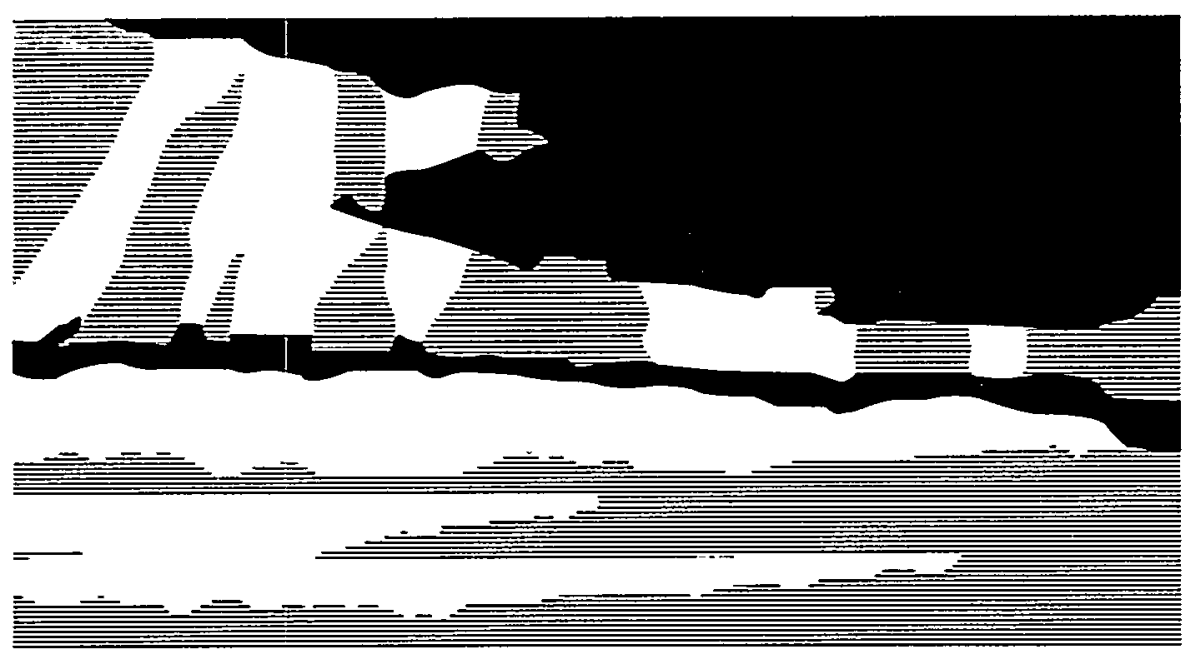

1180

$2890 \div 46$

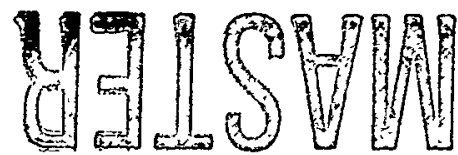

L66I Kienuef :aip

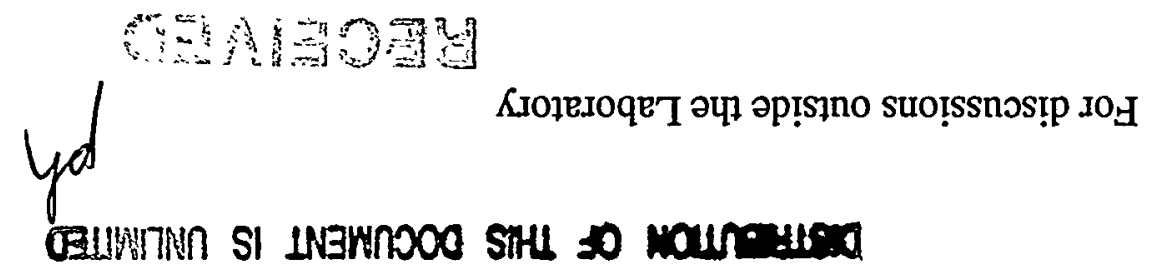

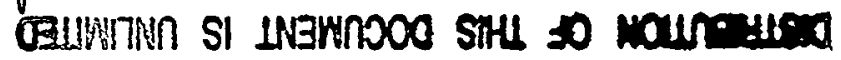

:01 poH!wquS

:(s)دouाn
T'HCOW XATH INGNOdWOD

-OML $V$ Y'GANA DOTVIVD

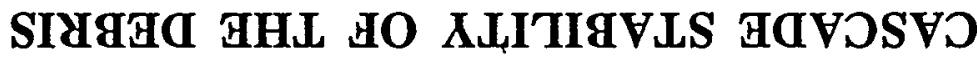




\section{DISCLAMERR}

Portions of this-docmment may- be illegible in electronic image products- Images:areproduced from the best available original dociment. 


\section{DISCLAIMER}

This report was prepared as an account of work sponsored by an agency of the United States Government. Neither the United States Government nor any agency thereof, nor any of their employees, make any warranty, express or implied, or assumes any legal liability or responsibility for the accuracy, completeness, or usefulness of any information, apparatus, product, or process disclosed, or represents that its use would not infringe privately owned rights. Reference herein to any specific commercial product, process, or service by trade name, trademark, manufacturer, or otherwise does not necessarily constitute or imply its endorsement, recommendation, or favoring by the United States Government or any agency thereof. The views and opinions of authors expressed herein do not necessarily state or reflect those of the United States Government or any agency thereof. 


\title{
CASCADE STABILITY OF THE DEBRIS CATALOG UNDER A TWO-COMPONENT FLUX MODEL
}

\author{
Gregory H. Canavan
}

\begin{abstract}
Stability is analyzed with a two component model. The critical densities derived are in agreement with other estimates in the appropriate limits. The current catalog is stable for nominal decay parameters, although further growth or cascade coefficients higher than nominal could be causes for concern. Sources reduce stability, which is more of a problem at high altitudes than low.
\end{abstract}

A two-component flux model, i.e., a model that treats the evolution of the fluxes of both large, intact objects and small fragments explicitly, has been shown to give reasonable predictions of the current catalog debris distribution and of the growth or decay of debris in coming decades and centuries. ${ }^{1}$ In the long term, an important issue is whether external sources or debris collisions could drive their distributions to a configuration in which collisions could produce cascade growth. The boundary at which debris production just balances loss by decay defines the "critical density" at each altitude. In the two-component model, that is the density at which the time derivatives of both the large objects and fragments equal zero. The flux model derived for predicting debris growth can be solved analytically for that condition, which determines the steady state density of both large objects and fragments at each altitude. These critical densities are in agreement with estimates by others in the appropriate one-component limit. For two independent components, they indicate the current catalog is stable for nominal decay parameters, although further growth, larger sources, or higher than nominal cascade coefficients could be causes for concern.

Two component results show that the catalog is strongly stable at $950 \mathrm{~km}$; less stable at $1,450 \mathrm{~km}$ and above for nominal parameters. If cascade coefficients proved larger than expected, the critical densities would be reduced correspondingly. External sources from launch, deployment, and explosions, which have produced essentially all of the current catalog, lower critical densities, making the catalog less stable at present and progressively less stable with time. The maximum sources that produce stable distributions are determined analytically. They produce critical densities about a factor of two below the cascade-driven densities, because adding external sources reduces the possible contribution from cascading, which lowers the steady state density at each altitude.

Current sources are generally above these critical sources, so densities should grow. However, the time scales for growth are long. At $950 \mathrm{~km}$, the catalog is very stable in the absence of sources, so eliminating them would recover strong stability. The reduction of the sources could be delayed for several centuries, and the catalog would still cease to grow when 
those sources were eliminated. At $1,450 \mathrm{~km}$, the catalog is marginally stable without sources and strongly unstable with them, so if sources remain at current levels, the boundary to instability should eventually be crossed. Eliminating the sources thereafter would no longer eliminate growth, which would then be driven by cascading. However, doubling times would be millennial and the total number of objects produced would be bounded.

Two-component flux model. The orbital decay model used below is an adaptation of one developed for stability calculations, which retains some measure of the eccentricity of the debris distribution but remains simple enough for understanding and parameter variations. ${ }^{2} \mathrm{~A}$ companion note describes how that model can be expanded to treat the both large, intact objects and small debris fragments and how the two eccentricities of the large objects and fragments can each be collapsed to a single density with an appropriate average lifetime. ${ }^{3}$ The resulting two equations for the two component densities at each altitude are the basic model used here. The model divides the atmosphere $<2,000 \mathrm{~km}$ into $100 \mathrm{~km}$ bins, and divides the number of particles in each altitude bin into large objects, $N$, and fragments, $n$, with the dividing line between them being about $0.5 \mathrm{~m}^{2.4}$ The densities of the large objects and fragments evolve under

$$
\begin{aligned}
& \mathrm{dN} / \mathrm{dt}=\mathrm{S}-\mathrm{N} / \mathrm{T}+\mathrm{F}_{+}+\mathrm{fQ}(\mathrm{N}+\mathrm{n})^{2}, \\
& \mathrm{dn} / \mathrm{dt}=\mathrm{s}-\mathrm{n} / \mathrm{t}+\mathrm{f}+(1-\mathrm{f}) \mathrm{Q}(\mathrm{N}+\mathrm{n})^{2},
\end{aligned}
$$

where the dependence of the equations on altitude, $z$, is suppressed, although such an equation holds for the two component densities in each $100 \mathrm{~km}$ altitude bin. The first term on the right hand side of each equation is the rate of production of fragments from launches and explosions, which are denoted by $S$ and $s$ for large objects and fragments, respectively. The second term represents decay at rates $1 / \mathrm{T}$ and $1 / \mathrm{t}$ for the large objects and fragments. The third is the flux of particles decaying from higher orbits, which is not important at the high altitudes of concern here, and is ignored below. The last is the collisional cascading rate for the total of $\mathrm{N}+\mathrm{n}$ particles and cascade coefficient $Q$, which is evaluated directly from the AFSPC debris catalog, 5 as is $f$, the fraction of objects produced by a collision that are large, which is about $f \approx 0.2$. Stability, or more properly steady state, is defined by the conditions $\mathrm{dN} / \mathrm{dt}=\mathrm{dn} / \mathrm{dt}=0$, for which Eqs. (1) and (2) can each be solved for

$$
\mathrm{Q}(\mathrm{N}+\mathrm{n})^{2}=(\mathrm{n} / \mathrm{t}-\mathrm{s}) /(1-\mathrm{f})=(\mathrm{N} / \mathrm{T}-\mathrm{S}) / \mathrm{f}
$$

from which

$$
\mathrm{n}=\mathrm{U}+\mathrm{VN}, \quad \text { t }
$$

where $R=(1-f) / f, U=(s-R S)$, and $V=R t / T$. This solution for $n$ in terms of $N$ can be substituted into Eq. (1) to obtäin

$$
N=\left[-b+\sqrt{ }\left(b^{2}-4 a c\right)\right] / 2 a,
$$

where $a=f Q(1+V)^{2}, b=-1 / T+2 f Q(1+V) U$, and $c=S+f Q U^{2}$. 
One-component limit. The zero source, single component, large object model corresponds to basing the analysis only on Eq. (1), and choosing $\mathrm{f}=1, \mathrm{R}=0$, and $\mathrm{s}=\mathrm{S}=0$, so that $\mathrm{U}=\mathrm{s}-\mathrm{RS}=0$, and $\mathrm{V}=0$. Those choices produce $\mathrm{n}=0$ and $\mathrm{a}=\mathrm{Q}, \mathrm{b}=-1 / \mathrm{T}, \mathrm{c}=0$, and

$$
\mathrm{N}=(1 / \mathrm{T}+1 / \mathrm{T}) / 2 \mathrm{Q}=1 / \mathrm{TQ} \text {. }
$$

In this nearly trivial limit, the only important parameter is the cascade coefficient $\mathrm{Q}$. Figure 1 shows the critical density $\mathrm{N}+\mathrm{n}$ together with the current debris catalog $\mathrm{N}_{\text {cat }}$ as functions of altitude for nominal cascade coefficients, zero sources $(S=s=0)$, and equal large object and fragment decay rates, $\mathrm{T}=\mathrm{t}=100$ years, i.e., all objects being treated as large objects. Only the potentially unstable region from $800 \mathrm{~km}$ to $1,600 \mathrm{~km}$ is shown. The top curve at low altitudes is the critical density, which is denoted by solid squares. At $850 \mathrm{~km}$, it has a value of about 1,900 . It falls sharply to about 800 objects at $950 \mathrm{~km}$; increases to about 1,000 objects by $1,050 \mathrm{~km}$; then falls exponentially - crossing the curve for the current catalog at about $1,400 \mathrm{~km}$.

As the critical density lies slightly below the catalog at $950 \mathrm{~km}$ and well below it at 1,450 $\mathrm{km}$ and above, this curve is interpreted by NASA as indicating instability in its publications, 6 with which Fig. 1 is in good agreement for this one component limit. However, those onecomponent results differ strongly with the two-component analysis below.

Two-component results. The two component model with zero sources corresponds to choosing $R=(1-f) / f, U=s-R S=0$, and $V=R t / T$, from which $n=(R t / T) N$ and $a \approx f Q[1+$ $\left.(\mathrm{R} t / \mathrm{T})^{2}\right], \mathrm{b}=-1 / \mathrm{T}, \mathrm{c}=0$, and

$$
\mathrm{N}=1 / \mathrm{Ta}=1 / \mathrm{TfQ}\left[1+(\mathrm{Rt} / \mathrm{T})^{2}\right],
$$

The choices $f=1 / 2$ and $t=T$ give the result $N=1 / T Q$ of Eq. (6). For $t=0$, it reduces to $1 / T f Q$, for which the critical density is increased by the reciprocal of the large object fraction, which is about 0.2 , the value used in constructing Fig. $2 .{ }^{7}$ Equation (7) holds for fragment areal densities from the $3 \mathrm{~kg} / \mathrm{m}^{2}$ used in Fig to $10-30 \mathrm{~kg} / \mathrm{m}^{2}$. The fragments are produced on orbit, their areal densities are not directly measurable, and are known only imprecisely. Thus, this lack of sensitivity to the detailed values of those areal densities is an advantage in interpretation. 8

Figure 2 shows the critical density for the nominal mix of large objects and small fragments. The catalog is as above, only the scale has changed. For the mix of large and small objects, the critical density starts at 8,500 at $850 \mathrm{~km}$, and falls somewhat more slowly. At 950 $\mathrm{km}, \mathrm{N}_{\text {crit }}$ is about 3,500, which is about 4 times larger than $\mathrm{N}_{\text {cat }}$, indicating that the catalog is strongly stable for this mix. The critical density falls gradually with altitude, but by $1,450 \mathrm{~km}$, it has fallen to only $10 \%$ larger than $\mathrm{N}_{\text {cat }}$. Thus, the catalog is stable there, but the margin is much smaller than at lower altitudes.

Model differences. The differences between Figs. 1 and 2 are significant. The former is interpreted as meaning that the current catalog is unstable around 950 and $1,450 \mathrm{~km}$. The two component results using the same parameters shows that the catalog is strongly stable at $950 \mathrm{~km}$ 
and slightly stable at $1,450 \mathrm{~km}$ and above. As the only difference between the two calculations is the inclusion of the fragments, which are a measured and important portion of the total density, it would appear that NASA's conclusion that the regions around 950 and $1,450 \mathrm{~km}$ are unstable is an artifact of basing their analysis on a singe component model for large objects only, which cannot be justified physically. When both of the two components, large objects and fragments, are included, the current catalog is stable for all altitudes.

Large objects and fragments are created in comparable numbers, and can participate with about equal effectiveness in collisions. However, the small fragments are more influenced by drag, so they are removed faster. That reduces the number of objects available for collisions, which increases the critical density needed for self-sustaining interaction. That is the basis for the greater stability of the two component model relative to that of the one component, large object only model discussed earlier.

Cascading. Figures 1 and 2 are constructed for nominal cascade rates. There is some question about these rates, as the experimental basis for them is limited and little evidence of collision on orbit. ${ }^{9}$ As shown in Eqs. (5)-(7), the critical density scales inversely on the cascade coefficient. If cascade coefficients proved larger than expected, the critical densities would be reduced correspondingly. At altitudes of interest, the cascade coefficients are at issue by about a factor of 2. Figure 3 shows the result of increasing the cascade coefficient by a factor of four in two-component calculations with $\mathrm{T}=100$ years and $\mathrm{t}=3$ years. The result looks much like Fig. 1 , because decreasing $t$ from 100 to 3 with $f=0.2$ increases $N_{\text {crit }}$ by about a factor of 5 , but increasing $Q$ by a factor of 4 reduces it by a factor of 4 , leaving it about at the curve for large objects only. The major concern is again the large peak at $1,450 \mathrm{~km}$.

An offsetting factor is the fact that fragment production is not local. Collisions scatter fragments onto orbits several hundred kilometers higher or lower than their point of origin. That averaging redistributes the fragments produced over a larger space, which reduces their density and collision rate at altitudes of concern. It has been suggested that this effect can be treated approximately by reducing the effective cascade coefficient by about a factor of three, 10 which would essentially recover Fig.. 2. Treating this non-local deposition of fragments would, however, involve coupling the nonlinear terms in Eqs. (1) and (2) between adjacent altitudes, which would make analytic solution difficult or impossible, so it is not pursued further here.

Realizability of postulated static critical densities. Figure. 4 shows the current catalog along with its large object and fragment components, according to the definitions used above. The large objects are about $25 \%$ of the total. Their contribution is concentrated in the lower peak at $950 \mathrm{~km}$; their contribution at $1,450 \mathrm{~km}$ is $10-15 \%$. Figure 5 shows the catalog, critical, large object, and fragment densities versus altitude. The large object density is most of the critical density. It is much larger than the fragment and catalog densities, which are of comparable 
magnitude to each other and an order of magnitude smaller than the total and large object critical densities. Thus, while these derived densities are by definition stable, i.e., time independent, it is not clear how the large object and fragment distributions could get to them from the present distributions, which have quite different relative magnitudes and structures. The gap is much smaller at $1,450 \mathrm{~km}$, although there the critical density still implies a large object density about an order of magnitude larger than the small object density, while in the current catalog, those proportions are reversed.

Sources. While the distributions of Fig. 2 are stable, the catalog shown is that of today. The catalog is continuing to grow in time. If the debris sources remain distributed in the future as they have been in the past, the catalog will rise, reach, and cross the critical density. While there is a large gap between the catalog and critical density at $950 \mathrm{~km}$ that could take centuries to close, the gap at $1,450 \mathrm{~km}$ is small, and the catalog is also growing rapidly there. There appears to be a temporary respite due to the cessation of Soviet Launches to that altitude, but if they are resumed, the time for closure could be decades.

The calculations above ignore debris sources other than collisional cascading. There are also sources from launch, deployment, and explosions, which have produced essentially all of the current catalog. While the analysis of the full catalog is complicated, a significant simplification results from the recognition that the fraction of large objects from an explosion is about the same as the distribution from a collision; thus $R \approx s / S$, which means $U \approx 0$ and $V=R t / T=s t / S T$, the ratio of the steady state densities of fragments and large objects when these sources dominate collisions. These values give $n=(R t / T) N$ and $a \approx f Q\left[1+(R t / T)^{2}\right], b=-1 / T, c=S$, which produce

$$
\mathrm{N}=\left\{1 / \mathrm{T}+\sqrt{[}\left[1 / \mathrm{T}^{2}-4 \mathrm{fQ}\left(1+(\mathrm{Rt} / \mathrm{T})^{2}\right) \mathrm{S}\right]\right\} / 2 \mathrm{fQ}\left[1+(\mathrm{Rt} / \mathrm{T})^{2}\right] \text {. }
$$

For $\mathrm{t} \ll<\mathrm{T}$, this reduces to

$$
\mathrm{N}=\left\{1 / \mathrm{T}+\sqrt{ }\left[1 / \mathrm{T}^{2}-4 \mathrm{fQS}\right]\right\} / 2 \mathrm{fQ},
$$

which shows that including external sources decrease the critical density, because they leave less production from collisional cascading for a given rate of orbital decay.

The largest source that permits a steady state solution is determined by setting the determinant $b^{2}-4 a c$ in Eq. (8) to zero, which gives

$$
\mathrm{S}=1 / 4 \mathrm{fQT}^{2}\left(1+(\mathrm{Rt} / \mathrm{T})^{2}\right),
$$

shown in Fig. 6. The critical source from Eq. (10) is shown by the open squares, which range from $\approx 4 / \mathrm{yr}$ at $850 \mathrm{~km}$ to $0.005 / \mathrm{yr}$ at $1,550 \mathrm{~km}$. The dominant scaling is on $\mathrm{T}^{2}$, which means the critical source falls exponentially with a scale height about half that of the atmosphere, or $\approx 120$ $\mathrm{km}$. The actual current sources are shown by the top curve denoted by the solid squares. They start at about $5 / \mathrm{yr}$ at $850-950 \mathrm{~km}$, drop to $0.7 / \mathrm{yr}$ at $1,150-1,350 \mathrm{~km}$, and then rise to $2-3 / \mathrm{yr}$ at $1,450-1,550 \mathrm{~km}$. 
The current sources are above the critical sources, which means the densities will continue to grow as long as those sources are imposed. The critical and actual sources are fairly close at $850 \mathrm{~km}$, which means reducing the sources slightly would eliminate growth there. However, the gap between them is an order of magnitude at $950 \mathrm{~km}$, which means for stability, a 10-fold reduction would be required there as well as at $1,150-1,300 \mathrm{~km}$. At $1,450 \mathrm{~km}$, the sources would have to be reduced by about a factor of 100 to prevent growth.

Substituting the critical source of Eq. (10) into the density of Eq. (8) produces the steady state density sustained by both external sources and cascading

$$
\mathrm{N}=1 / 2 \mathrm{fQT}\left[1+(\mathrm{Rt} / \mathrm{T})^{2}\right] \text {. }
$$

which is about $1 / 2$ the critical density of Eq. (7) based on cascading only. As expected, adding external sources reduces the possible contribution from cascading, which lowers the steady state value of $\mathrm{N}$ at each altitude.

Growth rates. While this note primarily discusses static solutions, some observations can be made about growth rates for large external sources. At $950 \mathrm{~km}$, the current sources are about an order of magnitude above the critical sources, which means the density would grow at about a net rate of $6 / y r$. The catalog is about 1,000 objects there, so the source-driven doubling time would be about 1,000 objects / 6 objects/yr $\approx 170$ years. That is to be contrasted to cascade growth, which has a coefficient of $\approx 10^{-} 6 \%$ object-yr, here, which gives a doubling time of $\approx$ $1 /\left(1,000 \times 10^{-6} \%\right.$ object-yr $) \approx 1,000$ years. For fixed sources, the debris growth would be dominated by external sources for a number of centuries before cascading became significant.

At $1,450 \mathrm{~km}$ there are about 600 catalog objects, and the source rate is about $3 / \mathrm{yr}$, so the external source doubling time would be about 200 years and the cascade doubling time $\approx 1 /(600$ $\times 3 \times 10^{-7} /$ object-yr) $\approx 6000$ years. Thus, current sources would not produce dangerous levels of debris for $\approx 10,000 / 3 / \mathrm{yr} \approx 3,000$ years, and cascading would not become significant for about half that interval. Thus, while the region at $1,450 \mathrm{~km}$ is unstable to current sources and difficult to stabilize without eliminating those sources entirely, the time scales for externally supported and cascade growth are quite long there.

The addition of external sources lowers critical densities, making the catalog less stable at present and progressively less stable with time. However, current sources are such that the time scales for source-supported and cascade growth are long. These issues are manifested in different ways at low and high altitudes. Around $950 \mathrm{~km}$, the catalog is very stable in the absence of sources, so eliminating them would recover strong stability even at higher catalog debris levels. That means the reduction of debris could be delayed for several centuries, and the $\approx 10 \%$ larger catalog would still cease to grow when those sources were eliminated.

Around $1,450 \mathrm{~km}$, the catalog is marginally stable without sources and strongly unstable with them. If the current sources are maintained there, the boundary to instability could be 
stability, even at higher catalog debris levels, which means the reduction of debris could be delayed for several centuries, and the catalog would still cease to grow when those sources were eliminated. At $1,450 \mathrm{~km}$, the catalog is marginally stable without sources and strongly unstable with them, so if sources remained at current levels, the boundary to instability should eventually be crossed. Eliminating the sources thereafter would no longer eliminate growth, which would then be driven by cascading. However, the doubling times would be millennial and the total number of objects produced would be bounded. Eliminating the sources at high altitudes soon would maintain the marginal stability there.

\section{References}

1. G. Canavan, "Estimates of Current Debris from Flux Models," Los Alamos report, August 1996.

2. D. Kessler, "Collisional Cascading: The Limits of Population Growth in Low Earth Orbit," Adv. Space Res, Vol. 11, 1991, p. (12)63.

3. G. Canavan, "Estimates of Current Debris from Flux Models," op. cit.

4. G. Canavan, "Fraction of Space Debris Collisions that are catastrophic," Los Alamos report, LA-UR-96-2802.

5. G. Canavan, "Rates Inferred from the Space Debris Catalog," Los Alamos report, LA-UR-962803.

6. D. Kessler, "Collisional Cascading: The Limits of Population Growth in Low Earth Orbit," op. cit., Fig. 4.

7. G. Canavan, "Estimates of Current Debris from Flux Models," op. cit.

8. G. Canavan, "Averaging of Decay Times and Rates over Debris Spectrum," Los Alamos report, LA-UR-96-2457.

9. D. McKnight, et. al., "Fragmentation Algorithms for Strategic and Theater Targets (FASTT) Empirical Breakup Model," DNA-Kaman January 1994.

10. D. Kessler, "Collisional Cascading: The Limits of Population Growth in Low Earth Orbit," op. cit. 
crossed. Eliminating the sources thereafter would no longer eliminate growth, as it would then be driven by cascading, although the doubling times would be millennial and the total number of objects produced would be bounded. Eliminating the sources at high altitudes soon would at best maintain the marginal stability there. To be effective, the reduction would have to be implemented promptly, and the catalog might continue to grow slowly there even then, because of the uncertainties in the models discussed above.

Summary and conclusions. An important long-term issue is whether debris sources could produce a debris distribution in which collisions could produce cascade growth. A twocomponent model gives reasonable predictions of the current catalog debris distribution, the growth or decay of debris in coming decades and centuries, and the stability of debris configurations. For useful predictions, at least a two-component treatment is essential. The model used retains some measure of the eccentricity of the debris distribution, but is expanded to treat both large, intact objects and small debris fragments through single densities with appropriate average lifetimes. The critical densities derived in the one-component limit are in agreement with other estimates.

Two component results show that the catalog is strongly stable at $950 \mathrm{~km}$; less stable at $1,450 \mathrm{~km}$ and above. NASA's conclusion that the regions around 950 and $1,450 \mathrm{~km}$ are unstable appears to be an artifact of their use of a singe component model for large objects only in their analysis. The critical density scales inversely on the cascade coefficient. If cascade coefficients proved larger than expected, the critical densities would be reduced correspondingly. The maximum expected variation in this altitude region is about a factor of two, which would impact stability at $1,450 \mathrm{~km}$ but not at $950 \mathrm{~km}$. This sensitivity is apparently offset by the non-locality of the fragment deposition. The realizability of postulated static critical densities is unclear, as they involve much larger fractions of large objects than are present in the current catalog.

The addition of external sources lowers critical densities, making the catalog less stable at present and progressively less stable with time. External sources such as objects from launch, deployment, and explosions have produced essentially all of the current catalog. Their analysis is simplified by the recognition that the fraction of large objects from explosions is about the same as that from collisions. The maximum source that is stable at each altitude can be determined analytically, and depends inversely on the product of the fraction of large objects, the cascade coefficient, and the square of the large object decay time. Those sources produce critical densities a factor of two below the cascade-driven densities, as adding external sources reduces the possible contribution from cascading, which lowers the steady state density at each altitude.

Current sources are generally above these critical sources, so densities should grow. However, the time scales for source-supported and cascade growth are long. At $950 \mathrm{~km}$, the catalog is very stable in the absence of sources, so eliminating them would recover strong 


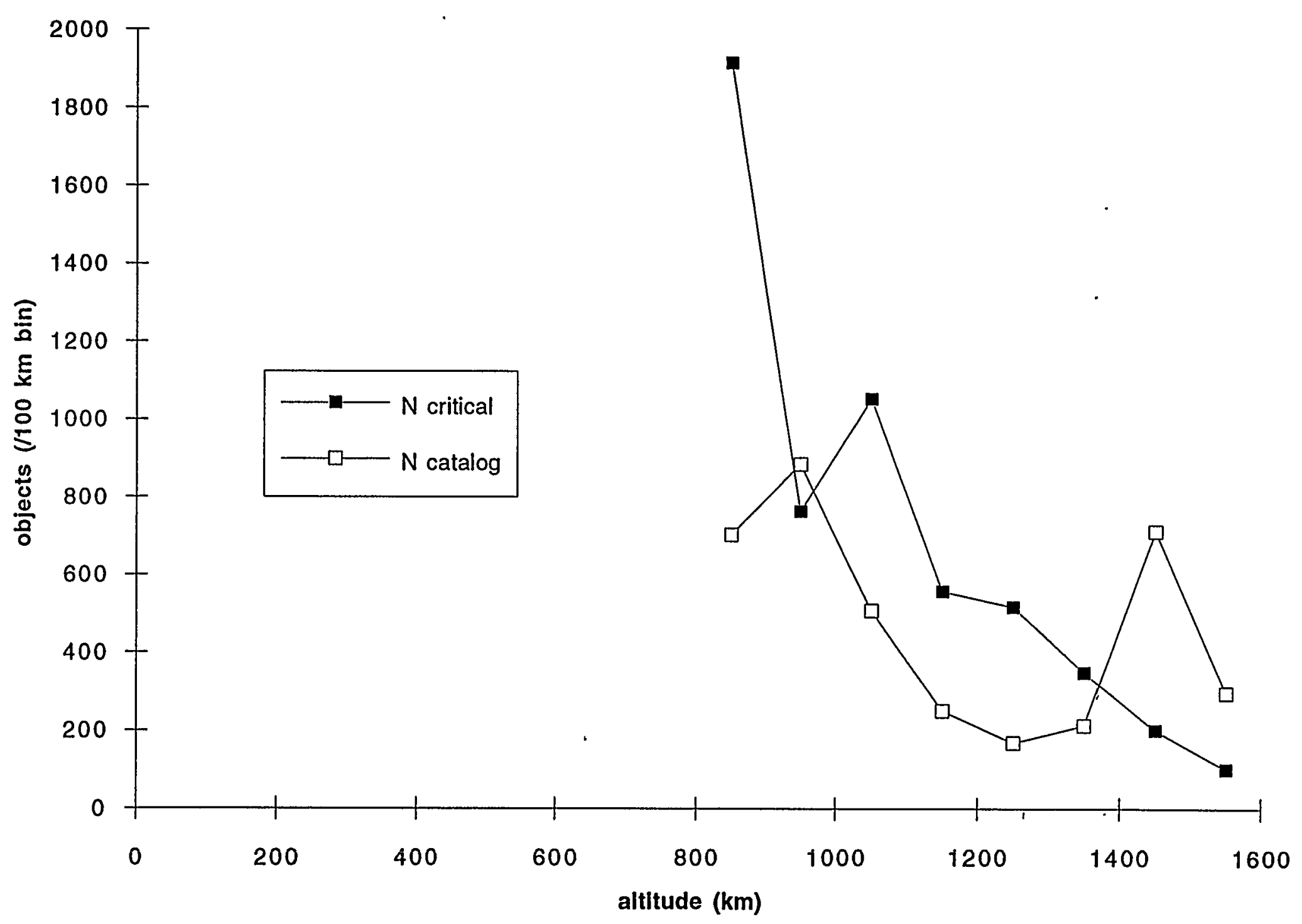

Fig. 1. Current catalog and critical object densities for large objects plus fragments versus altitude. 


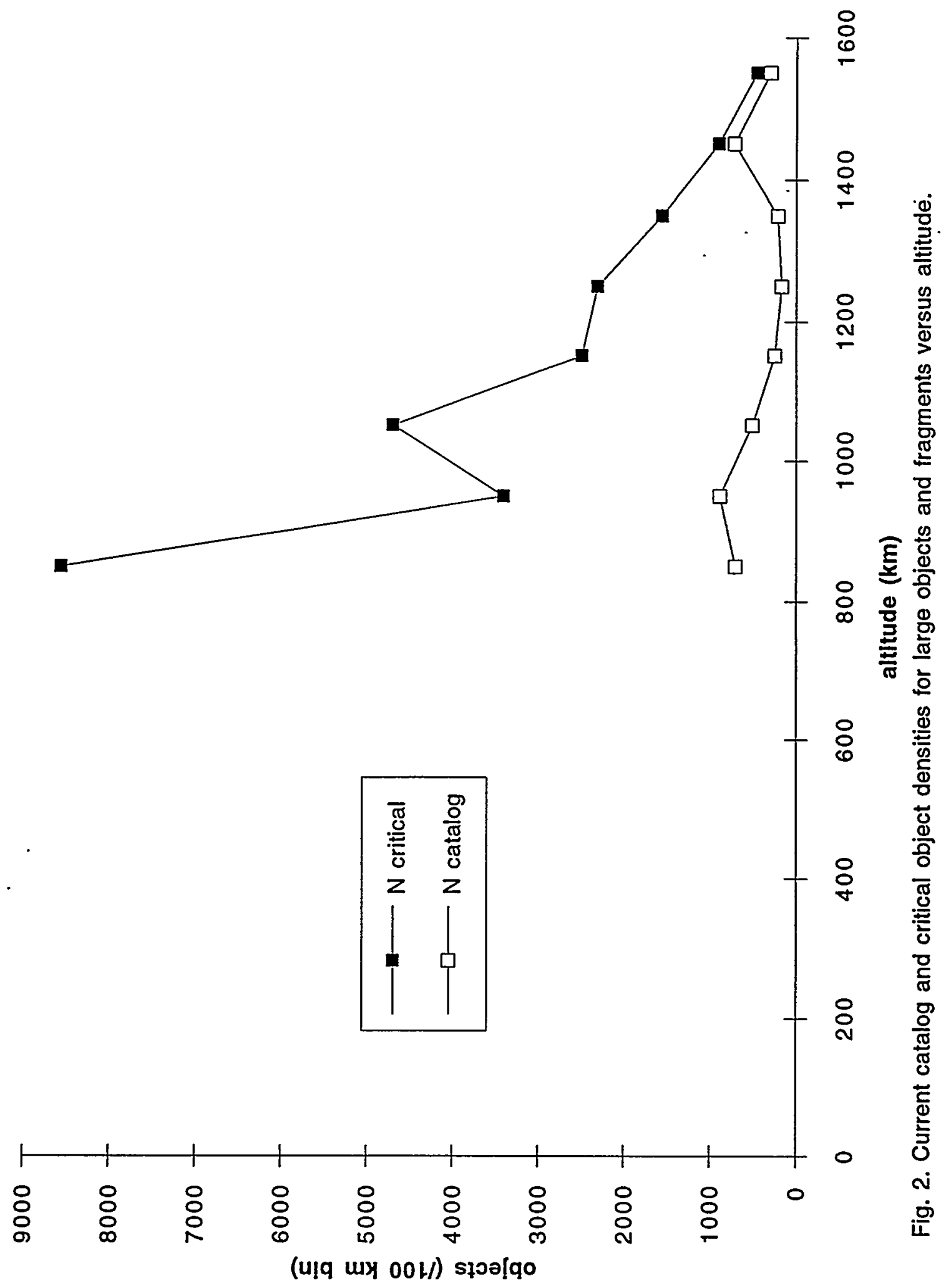




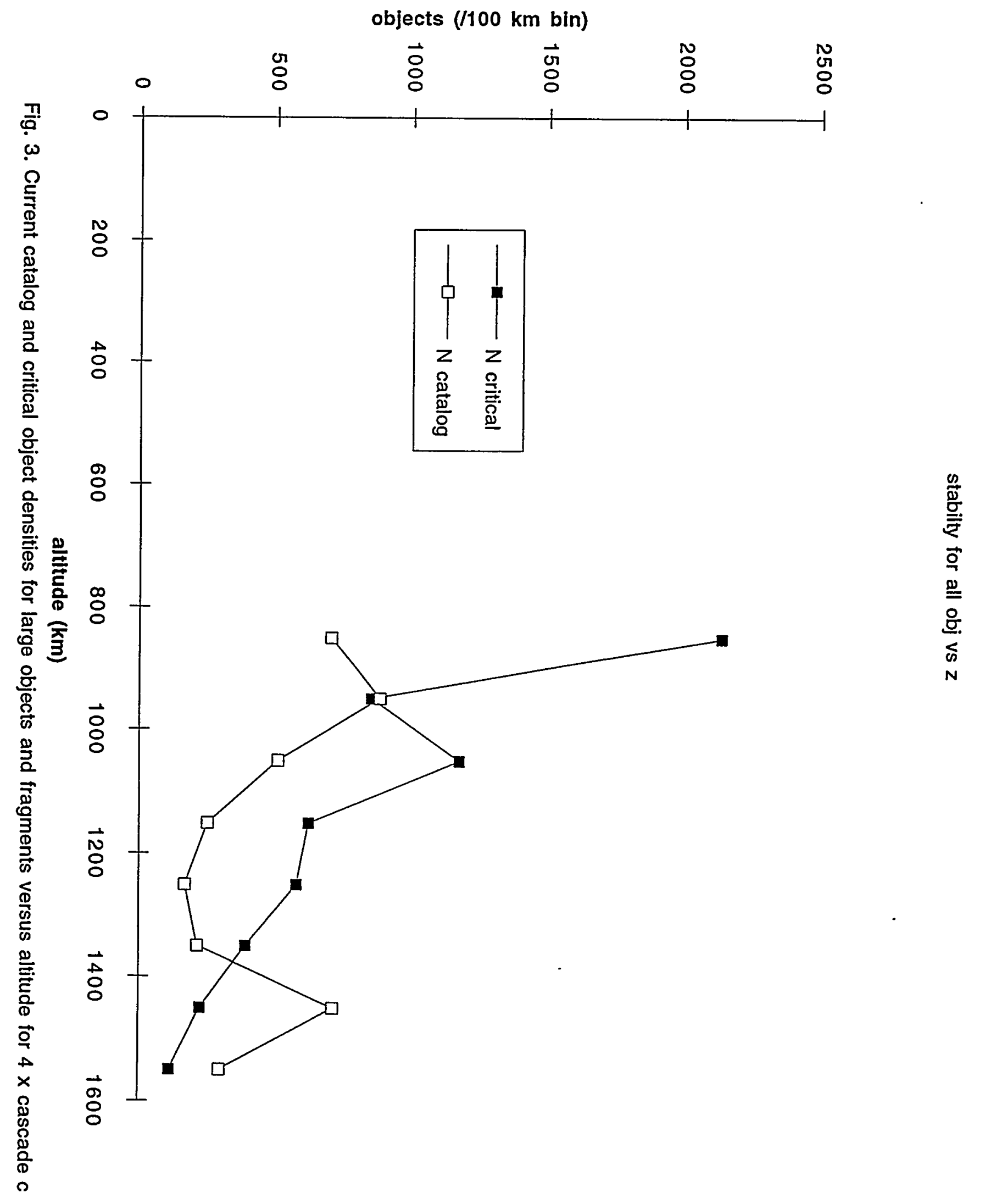



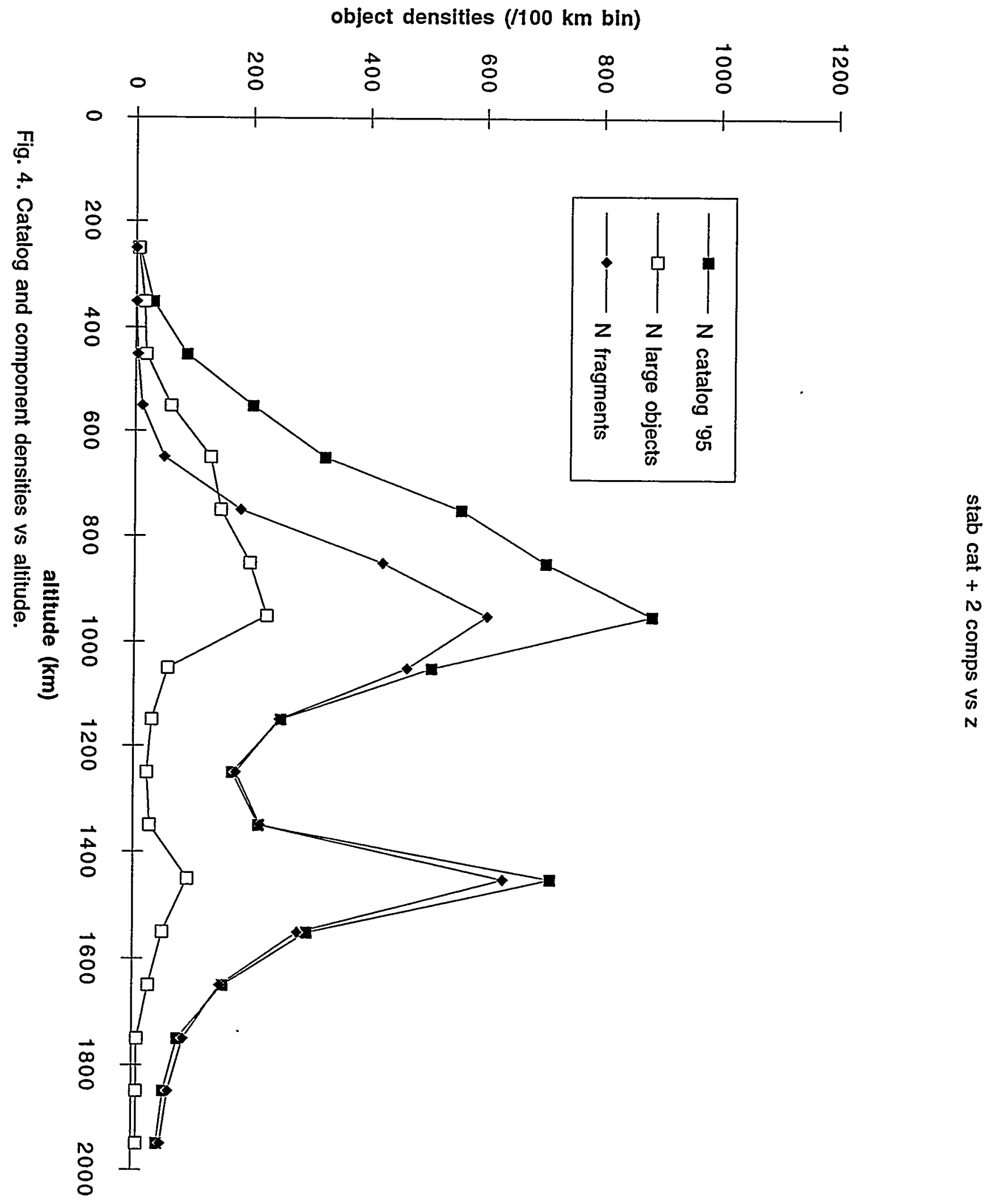


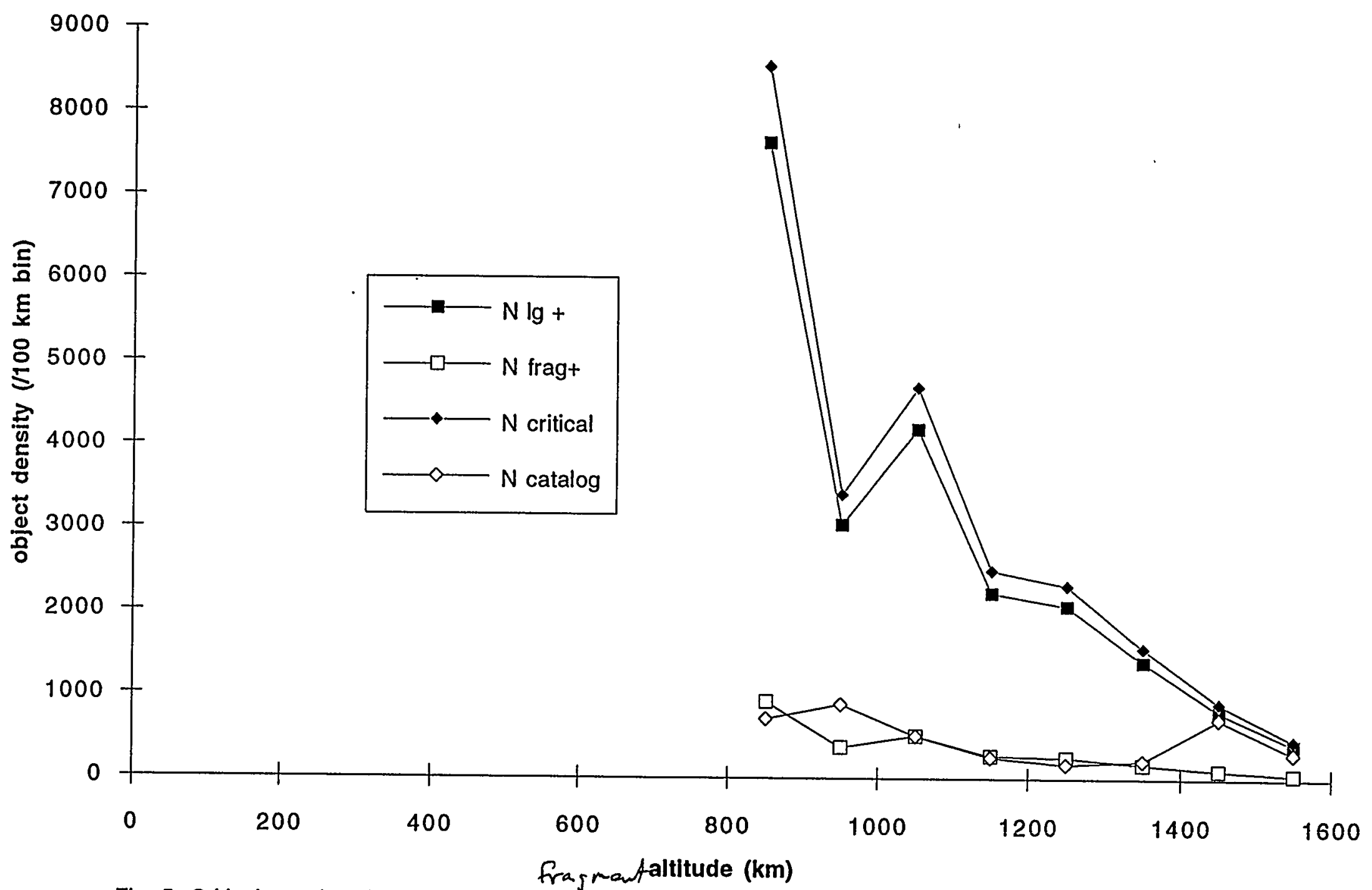

Fig. 5. Critical, catalog, large object, and eatatog density versus altitude. 


\section{(แя) әрпџ!|एе}

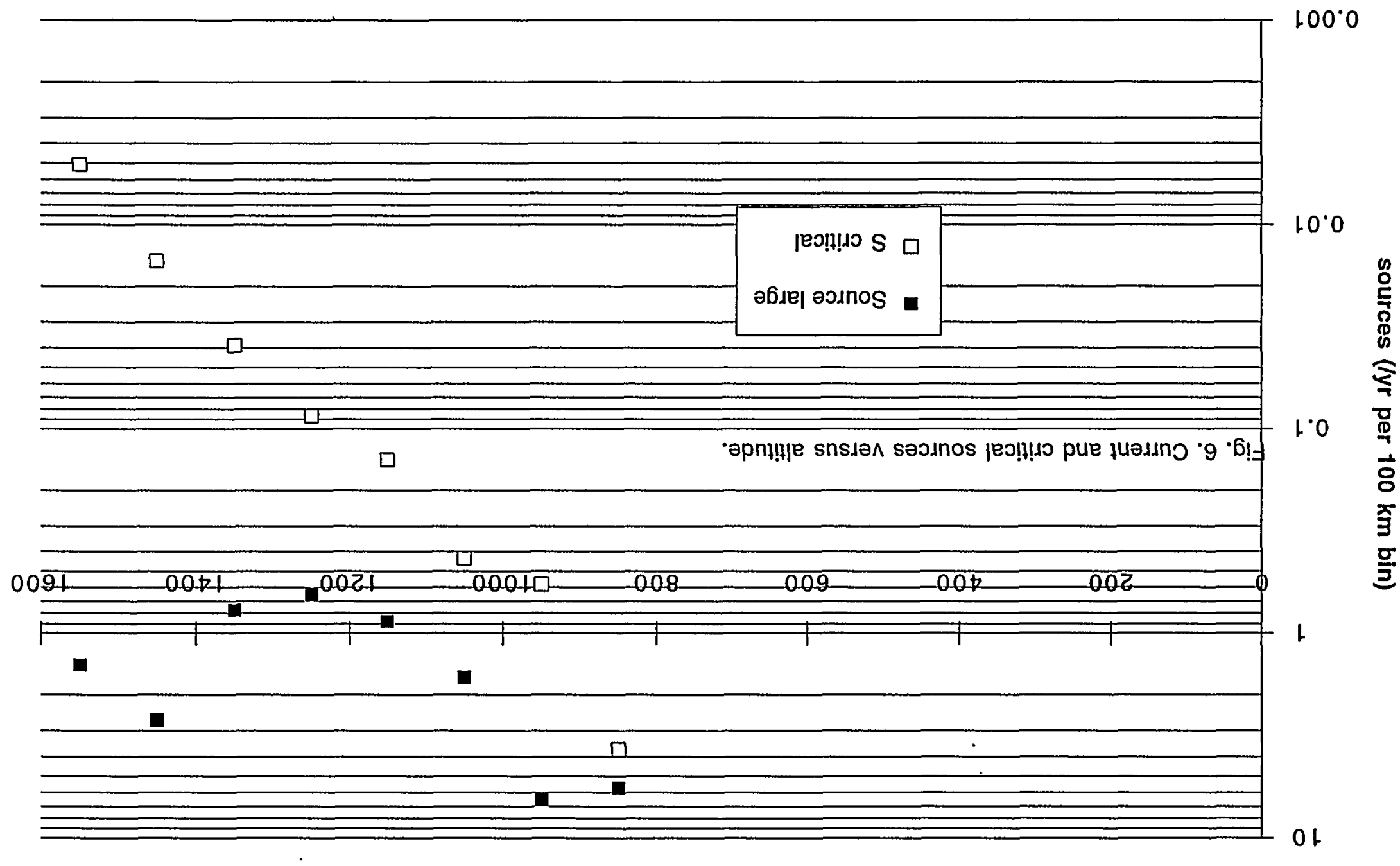

$z$ s^ $0=1001$ 10। องมnos 6 